Health in Lisbon and Lung Diseases Centre of Venda Nova, Amadora, Portugal. ${ }^{\S \S}$ TB Clinic, Dept of Ambulatory Care and Community Medicine, University of Lausanne, Lausanne, Switzerland. ${ }^{f f}$ Scientific Advice Unit, European Centre for Disease Prevention and Control, Stockholm, Sweden.

Correspondence: G.B. Migliori, World Health Organization Collaborating Centre for Tuberculosis and Lung Diseases, Fondazione S. Maugeri, Care and Research Institute, Via Roncaccio 16, 21049, Tradate, Italy. E-mail: giovannibattista. migliori@fsm.it

Support Statement: The research leading to this publication has been performed under the European Centre for Disease Prevention and Control (ECDC) service contract 1757 adjudicated to TBNET following open tender competition.

Statement of Interest: None declared.

\section{REFERENCES}

1 World Health Organization. Multidrug and Extensively DrugResistant TB (M/XDR-TB). 2010 Global Report on Surveillance and Response. WHO/HTM/TB/2010.3. Geneva, World Health Organization, 2010.

2 Sotgiu G, Ferrara G, Matteelli A, et al. Epidemiology and clinical management of XDR-TB: a systematic review by TBNET. Eur Respir J 2009; 33: 871-881.

3 Migliori GB, Loddenkemper R, Blasi F, et al. 125 years after Robert Koch's discovery of the tubercle bacillus - the new XDR-TB threat.
Is "science" enough to tackle the epidemic? Eur Respir J 2007; 29 423-427.

4 Migliori GB, Besozzi G, Girardi E, et al. Clinical and operational value of the extensively drug-resistant tuberculosis definition. Eur Respir J 2007; 30: 623-626.

5 Migliori GB, Ortmann J, Girardi E, et al. Extensively drug-resistant tuberculosis, Italy and Germany. Emerg Infect Dis 2007; 13: 780-782.

6 World Health Organization. Extensively drug-resistant tuberculosis (XDR-TB): recommendations for prevention and control. Wkly Epidemiol Rec 2006; 81: 430-432.

7 Raviglione MC, Uplekar MW. WHO's new Stop TB Strategy. Lancet 2006; 367: 952-955.

8 World Health Organization. WHO Policy on Infection Control in Health-Care Facilities, Congregate Settings and Households. WHO/HTM/TB/2009.419. Geneva, World Health Organization, 2009.

9 Sotgiu G, Centis R, D'Ambrosio L, et al. Development of a standardised tool to survey MDR-/XDR-TB case management in Europe. Eur Respir J 2010; 36: 208-211.

10 Miravitlles M, Ferrara G, Lange C, et al. TB or not TB: update from the ERS Respiratory Infection Assembly 10. Eur Respir J 2010; 36: 665-670.

11 Tuberculosis Coalition for Technical Assistance. International Standards for Tuberculosis Care (ISTC), 2nd Edn. The Hague, Tuberculosis Coalition for Technical Assistance, 2009.

12 Nardell E, Dharmadhikari A. Turning off the spigot: reducing drug-resistant tuberculosis transmission in resource-limited settings. Int J Tuberc Lung Dis 2010; 14: 1233-1243.

\title{
Effects of distance to treatment centre and case load upon tuberculosis treatment completion
}

\section{To the Editors:}

The prompt identification and adequate treatment of tuberculosis (TB) cases are key components of the global control effort [1]. In many high-income countries, TB is relatively uncommon, implying that many clinicians do not have regular and continuing experience of managing TB. To address this, a policy response is a trend towards small numbers of centralised treatment facilities where individuals may receive better and more complete treatment $[2,3]$. This centralising trend is the opposite to many low-income countries, where there is a move towards larger numbers of more decentralised TB services to facilitate patient access [4]. The difference is that such countries have sufficient cases to allow clinical staff to maintain their expertise, even in rural areas.

If $\mathrm{TB}$ services become more centralised, then patients may experience greater difficulty in accessing $\mathrm{TB}$ services due to increasing the distance between the home and treatment centre. There is little research on how distance affects TB completion, but rural residence is a known risk factor associated with a delay in the diagnosis and treatment of TB
[5] and may therefore affect treatment completion. However, most of this evidence comes from low-income countries. In contrast, in high-income countries, good transport links and reasonable social security systems imply that such barriers may not exist.

This epidemiological study examined whether treatment centre case load (annual number of TB patients seen) and healthcare accessibility (proximity of patient residence to TB treatment centre) have an influence upon the completion of TB treatment in England and Wales, UK.

The study was based upon a sample of 21,954 patients reported to the national enhanced TB surveillance system (ETS) from 2001 to 2006 in England and Wales. These TB patients all had their diagnosis confirmed by bacteriological culture or had clinical/radiological/histopathological features suggestive of $\mathrm{TB}$, and their clinician had taken the decision to treat the patient with a full course of anti-TB therapy. This sample was $50.3 \%$ of the total cases reported to the ETS during this period. Cases had to be excluded from the study mainly due to missing information on where the patient was actually treated 
for TB. This may have been different to the original clinic where TB was diagnosed. A comparison of all patients on the ETS within our study sample indicated close demographic similarities.

Treatment outcome was categorised as either completion or noncompletion based on clinical information 12 months after the date of notification or start of TB treatment (further details of ETS is provided in [6]). Patients with multidrug-resistant TB were excluded because current treatment regimens recommend a course of therapy of $\geqslant 18$ months.

For each patient, the experience of the TB centre where they were treated was obtained by calculating the number of cases seen by that centre for the year in which their case was notified. The median centre case load for patients was 71 cases $\cdot \mathrm{yr}^{-1}$ (interquartile range (IQR) 26-130 cases $\cdot \mathrm{yr}^{-1}$ ). For each patient, a measure of healthcare accessibility was calculated by obtaining the road distance from their home (based on postcode) to the TB treatment centre using a geographical information system. The majority of patients lived in close proximity to their treatment centre and the median road distance was $4.4 \mathrm{~km}$ (IQR 2.9-7.3 km).

For each patient, the potentially confounding variables age, sex, place of birth (country), number of years living in the UK, ethnicity, previous history of $\mathrm{TB}$, site of the disease and isoniazid susceptibility were obtained from the ETS. At the ecological level, confounding variables were also collected, specifically the 2004 index of multiple deprivation [7] and an urban rural classification [8]. The region of residence was obtained from the ETS.

The analysis proceeded in stages. The first stage produced a univariable model to examine the impact of all the potential explanatory variables upon completion of treatment. This was investigated using logistic regression to determine odds ratios for each of the explanatory variables. In these analyses, the TB centre load variable was dichotomised to $>26$ or $\leqslant 26$ cases per annum. The road distance to the TB treatment centre variable was also dichotomised to $>7.3$ or $<7.3 \mathrm{~km}$. These categories were decided upon by examining plots of treatment outcome against the individual variables. A multivariable model was then fitted including all of the individual confounding variables as well as the centre load and healthcare accessibility measures.

The results of the univariable and multivariable results indicated that attending a TB centre with a low case load was associated with poorer treatment outcomes $(\mathrm{p}<0.01)$ and travelling a greater distance to the TB treatment centre was associated with poorer treatment outcomes $(\mathrm{p}<0.001)$.

During these analyses, significant interactions were observed between both the centre load and healthcare accessibility variables with the variables measuring whether or not the patient was born in the UK. Significant interactions were also observed between the healthcare accessibility variables and the patient age variable. Therefore, in the final analysis, multivariable models were produced for UK- and non-UK-born patients separately, who were additionally subdivided into individuals $<45$ and $>44$ yrs of age. All the models were tested with and without the potentially confounding ecological variables (urban rural classification, index of multiple deprivation and region). The results presented were unaffected by the inclusion of these variables. Analysis was undertaken in STATA SE 8.0 (StataCorp, College Station, TX, USA).

The final results are presented in table 1, and it is important to emphasise that these are based upon a multivariable model that included a number of other variables listed in the table. These indicated that for both young and older individuals born in the UK, TB centre load was insignificant (OR $0.79(\mathrm{p}=0.05)$ and OR $1.13(\mathrm{p}=0.15)$, respectively). For individuals born outside the UK, TB centre load was significant for young individuals, indicating poorer completion of treatment at centres with low case loads (OR 0.75; $\mathrm{p}<0.001$ ). There was no effect on older individuals (OR 0.91; $\mathrm{p}=0.358$ ). Turning our attention to road distance to the TB treatment centre, for individuals born in the UK, this was insignificant for young and older individuals (OR $1.05(\mathrm{p}=0.68)$ and OR 0.94 $(p=0.446))$. For those born outside the $U K$, road distance to

Odds ratio (multivariable) analyses of factors affecting completion of tuberculosis (TB) cases in England and Wales, UK in 2001-2006 for young and older individuals born in and outside the UK

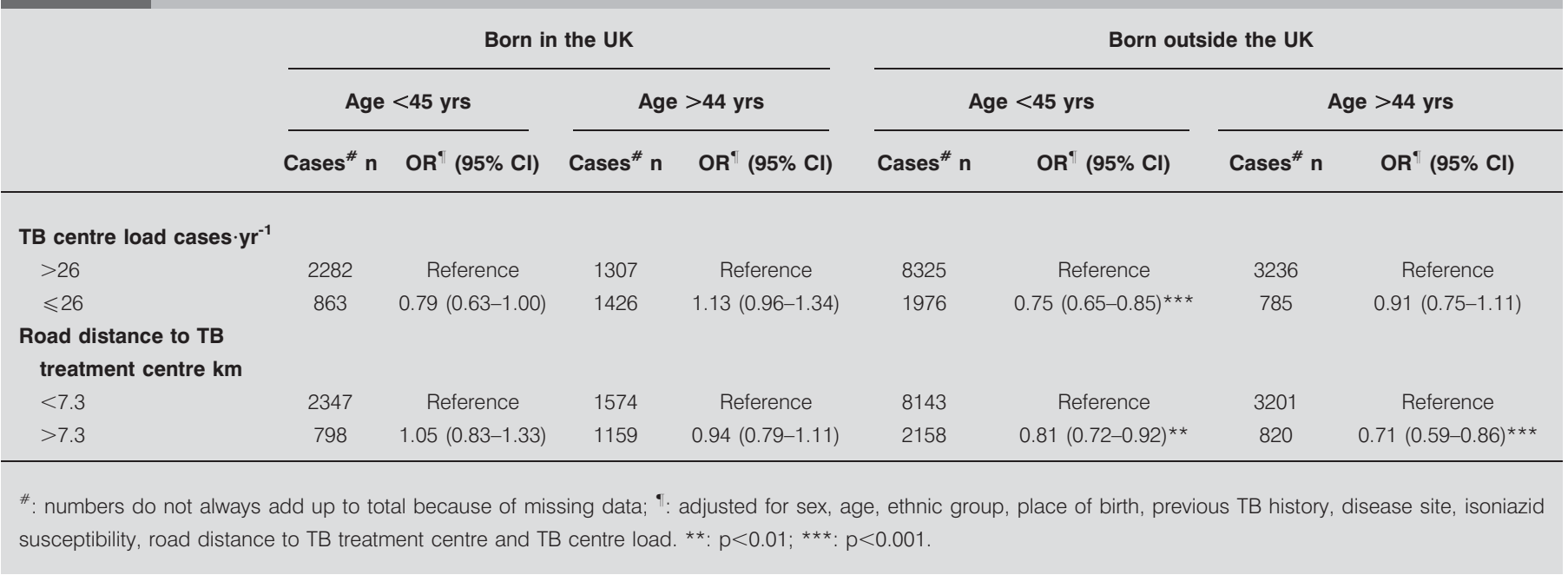


the TB treatment centre was significant for young and older individuals (OR $0.81 \quad(\mathrm{p}<0.01)$ and OR $0.71 \quad(\mathrm{p}<0.001)$, respectively), indicating lower completion of treatment if the patient lived $>7.3 \mathrm{~km}$ from the treatment centre.

$\mathrm{TB}$ is a rare disease in many high-income countries, which leads to pressures for the pooling of expertise to larger centres [2]. This results in patients being treated in TB centres with higher case loads but greater distances for patients to travel. This study demonstrates that such merging has positive health benefits, as larger centres (load $>26$ cases $\cdot \mathrm{yr}^{-1}$ ) had significantly higher completion rates for $\mathrm{TB}$ treatment for young individuals born outside the UK ( $\sim 50 \%$ of TB patients). However, if centralisation leads to patients having to travel greater distances to receive treatment, then this has negative consequences, as this study shows that all patients born outside the UK $(\sim 65 \%$ of TB patients) were less likely to complete treatment if they had to travel $>7.3 \mathrm{~km}$ to the TB treatment centre. Other studies have indicated the importance of provider experience to treatment outcome [3] but the present study is the first to demonstrate that such experience has a differing impact on subgroups of the population. Previous studies investigating delay in the diagnosis and treatment of $\mathrm{TB}$ have identified the importance of healthcare accessibility [5], but this is the first to be conducted in a high-income country. Additionally, it focuses upon treatment outcome as opposed to diagnosis delay, and examines differential effects amongst population subgroups.

Further research is needed to understand why young individuals born outside the UK are less likely to complete treatment at small TB centres. Migrants may face difficulties in accessing healthcare due to language problems or poor understanding of the health system [3]. They may also require culturally appropriate services [9]. Young migrants may face further difficulties as they require tailored health information. Small TB centres may be unable to address such issues. Many migrants are materially deprived, which, alongside language difficulties [10], may explain why travelling short distances to $\mathrm{TB}$ treatment centres appears difficult. This result was unexpected due to the high-income country setting and the fact that most patients lived close to their TB treatment centre.

This research indicates that moves towards centralisation of TB services, such as implementation of the UK recommendations that clinicians see $\geqslant 10$ TB patients per year [2], should be implemented alongside measures to ensure accessibility of services, targeted to individuals born outside the UK who live far from the treatment centres.

\section{I.R. Lake*, N.R. Jones*, L. Bradshaw" and I. Abubakar",}

*School of Environmental Sciences and "Norwich Medical School, University of East Anglia, Norwich, and "Tuberculosis Section, Centre for Infections, Health Protection Agency, London, UK.

Correspondence: I.R. Lake, School of Environmental Sciences, University of East Anglia, Norwich, UK. E-mail: i.lake@uea. ac.uk

Statement of Interest: None declared.

\section{REFERENCES}

1 WHO. The Stop TB Strategy. Report No. WHO/HTM/TB/ 2006.368. Geneva, World Health Organization, 2006.

2 Dept of Health. Tuberculosis Prevention and Treatment: a Toolkit for Planning, Commissioning and Delivering High-Quality Services in England. London, Dept of Health, 2007.

3 Gardam M, Verma G, Campbell A, et al. Impact of the patientprovider relationship on the survival of foreign born outpatients with tuberculosis. J Immigr Minor Health 2009; 11: 437-445.

4 Wei X, Liang X, Liu F, et al. Decentralising tuberculosis services from county tuberculosis dispensaries to township hospitals in China: an intervention study. Int J Tuberc Lung Dis 2008; 12: 538-547.

5 Storla DG, Yimer S, Bjune GA. A systematic review of delay in the diagnosis and treatment of tuberculosis. BMC Public Health 2008; 8:15.

6 Abubakar I, Crofts JP, Gelb D, et al. Investigating urban-rural disparities in tuberculosis treatment outcome in England and Wales. Epidemiol Infect 2008; 136: 122-127.

7 Office for National Statistics. Indices of Deprivation Across the UK. Newport, ONS, 2009.

8 Bibby P, Shepherd J. Developing a New Classification of Urban and Rural Areas for Policy Purposes. www.statistics.gov.uk/ geography/downloads/Methodology_Report.pdf Date last updated: 2001.

9 Eziefula C, Brown M. The health of recent migrants from resourcepoor countries. Medicine 2010; 38: 60-65.

10 Derose KP, Escarce JJ, Lurie N. Immigrants and health care: sources of vulnerability. Health Aff 2007; 26: 1258-1268.

DOI: 10.1183/09031936.0003621

\section{Tuberculosis-specific T-cell response after recent treatment and remote cure}

\section{To the Editors:}

Tuberculosis (TB) remains a major global health problem. A major factor contributing to the current TB epidemic is the resistance of Mycobaterium tuberculosis to first-line anti-TB treatment. Therefore, there is a need for an appropriate and reliable tool to evaluate treatment efficacy.

Several studies using the T-SPOT®.TB assay (Oxford Immunotec Ltd, Oxford, UK) reported a decline in the number of 